\title{
TO INVESTIGATE THE SOLUSION OF BIANCHI TYPE I STRING MODELS WITH DARK ENERGY IN BIMETRIC THEORY OF GRAVITATION
}

\author{
Vijay Lepse \\ Department of Mathematics, Science College Pauni-441910 \\ Abstract:- \\ The purpose of this research note is to know that the screech of the nature by evaluating cosmological model of \\ universe with dark energy in biometric theory of gravitation by using Rosen's field equations
}

\section{Introduction}

The latest discovery of modern cosmology is that the current universe is expanding and accelerating. The data base like Cosmic Microwave Background Radiation (CMBR) and such as Type Ia supernova (S Ne Ia) observed by Riess et al. (2004), Eisenstein et al. (2005), Astier et al. (2006) and Spergel et al. (2007) indicate that the universe containing only $4 \%$ ordinary baryonic matter, $23 \%$ dark matter and $73 \%$ dark energy. The dark matter is unknown form of matter which has clustering properties of ordinary matter and has not been yet detected. The dark energy is the term represents for an unknown form of energy which has not also been detected. The observations of Wilkinson Microwave Anisotropic Probe (WMAP) satellite experiment studied by Bennett et al. (2003), Spergel et al. (2003) and Sloan Digital Sky survey (SDSS) discussed by Perlmutter et al. (1998), Knop et al. (2003), Spergel et al. (2003, 2007), Abazajian et al. (2003, 2004), Tegmark et al. (2004), Seljack et al. (2005), Adelman et al. (2005), Komatsu et al. (2009, 2011) and Hinshaw (2013) [WMAP collaboration] and Ade et al. (2013) [Planck Collaboration] have proved that our universe is undergoing an accelerating expansion. The various forms of dark energy such as quintessence discussed by Ratra et al. (1988), Wetterich (1988), Liddle et al. (1999) and Zlatev et al. (1999) and phantom field (scalar field with negative sign of kinetic term) studied by Cadwell et al. (2002, 2003), Nojiri et al. (2003), Onemli et al. (2004) and Setare et al. (2008) and also the combination of quintessence have been studied in a unified model by Feng et al. (2005), Zhao et al. (2006), Setare et al. (2008) and Sadghi et al. (2008). Another type of dark energy Chaplygin gas model have been studied by Kamenshchik et al. (2001), Setare (2007, 2009) and Katore et al. (2011).

The equation of state parameter $\omega(t)$ has an important role in dark energy models. The constant values of $\omega=-1,0,+1 / 3$ and +1 represents vacuum fluid, dust, radiation and stiff dominated universe. The variable $\omega(t)$ of time or red shift is taken by researchers like Jimenez (2003) and Das et al. (2005) etc. The quintessence model, $\omega>-1$ (explanation of observations of accelerating universe) involving scalar field and phantom model $\omega<-1$ (expansion of universe increases to infinite degree in finite time) gives rise to time dependant parameter $\omega(t)$, which has been taken into account by Turner et al. (1997), Caldwell et al. (1998) and Liddle et al. (1999). Various forms of parameter $\omega(t)$ have been used for dark energy models and a binary mixture of perfect fluid and dark energy have been considered by many researchers like Steinhardt et al. (1999), Saha (2005, 2006), Rahman et al. (2006, 2009), Mukhopadhyay et al. (2008), Singh et al. (2009), Ray et al. (2010), Akarasu et al. (2010), Sharif et al. (2010), Yadav et al. (2011) and Pradhan et al. (2011) to investigate the cosmological models of the universe and discuss the different geometrical and physical aspects of the models.

\section{Metric and Rosen's Field Equations}

We consider the Bianchi Type I metric

$d s^{2}=-d t^{2}+A^{2} d x^{2}+B^{2} d y^{2}+C^{2} d z^{2}$

where $A, B$ and $C$ are the functions of $t$ only. 
The flat metric corresponding to metric (5.2.1) is

$d \eta^{2}=-d t^{2}+d x^{2}+d y^{2}+d z^{2}$

The energy momentum tensor $T_{i}^{j}$ of the source of bulk viscous fluid with string is given by

$$
T_{i}^{j}=\in v_{i} v^{j}-\lambda x_{i} x^{j}-\xi v_{\mid l}^{l}\left(g_{i}^{j}+v_{i} v^{j}\right)
$$

with

$$
v_{i} v^{j}=-x_{i} x^{j}=-1
$$

and

$$
v^{i} x_{i}=0
$$

Here $\in$ is the rest energy density and $\epsilon=\epsilon_{p}+\lambda$ where $\epsilon_{p}$ and $\lambda$ denote the particle density (particles loaded on the string) and the string tension density respectively, $x^{i}$ is the direction of string, $v^{i}$ is the four velocity vector, $\xi$ is the coefficient of bulk viscosity and $\theta$ is the scalar expansion which is given by

$$
\theta=v_{\mid l}^{l}
$$

Thus, the Rosen's field equations are,

$$
-\frac{\ddot{A}}{A}+\frac{\ddot{B}}{B}+\frac{\ddot{C}}{C}+\frac{\dot{A}^{2}}{A^{2}}-\frac{\dot{B}^{2}}{B^{2}}-\frac{\dot{C}^{2}}{C^{2}}=16 \pi A B C(\lambda+\xi \theta)
$$

$\frac{\ddot{A}}{A}-\frac{\ddot{B}}{B}+\frac{\ddot{C}}{C}-\frac{\dot{A}^{2}}{A^{2}}+\frac{\dot{B}^{2}}{B^{2}}-\frac{\dot{C}^{2}}{C^{2}}=16 \pi A B C \xi \theta$

$\frac{\ddot{A}}{A}+\frac{\ddot{B}}{B}-\frac{\ddot{C}}{C}-\frac{\dot{A}^{2}}{A^{2}}-\frac{\dot{B}^{2}}{B^{2}}+\frac{\dot{C}^{2}}{C^{2}}=16 \pi A B C \xi \theta$

$\frac{\ddot{A}}{A}+\frac{\ddot{B}}{B}+\frac{\ddot{C}}{C}-\frac{\dot{A}^{2}}{A^{2}}-\frac{\dot{B}^{2}}{B^{2}}-\frac{\dot{C}^{2}}{C^{2}}=16 \pi A B C \in$

From differential equations, we write

$\frac{d}{d t}\left(\frac{\dot{B}}{B}\right)=\frac{d}{d t}\left(\frac{\dot{C}}{C}\right)$

which on integrating, we get

$$
B=a_{1} C
$$

where $a_{1}$ is constant of integration and choosing the constant of integration $a_{1}=1$, so that $B=C$

Using equation (2.11), the differential equations can be written as

$$
-\left(\frac{\ddot{A}}{A}-\frac{\dot{A}^{2}}{A^{2}}\right)+2\left(\frac{\ddot{B}}{B}-\frac{\dot{B}^{2}}{B^{2}}\right)=16 \pi A B^{2}(\lambda+\xi \theta)
$$


$\left(\frac{\ddot{A}}{A}-\frac{\dot{A}^{2}}{A^{2}}\right)=16 \pi A B^{2} \xi \theta$

$\left(\frac{\ddot{A}}{A}-\frac{\dot{A}^{2}}{A^{2}}\right)+2\left(\frac{\ddot{B}}{B}-\frac{\dot{B}^{2}}{B^{2}}\right)=16 \pi A B^{2} \in$

On subtracting equations (2.14) and (2.15), we get

$-2\left(\frac{\ddot{A}}{A}-\frac{\dot{A}^{2}}{A^{2}}\right)+2\left(\frac{\ddot{B}}{B}-\frac{\dot{B}^{2}}{B^{2}}\right)=16 \pi A B^{2} \lambda$

Adding equations (2.15) and (2.16), we have

$-\left(\frac{\ddot{A}}{A}-\frac{\dot{A}^{2}}{A^{2}}\right)+4\left(\frac{\ddot{B}}{B}-\frac{\dot{B}^{2}}{B^{2}}\right)=16 \pi A B^{2}(\lambda+\epsilon)$

The conservation law for the energy-momentum tensor, yield

$\dot{\epsilon}=(-2 \in+\lambda) \frac{2 \dot{B}}{B}+\xi \theta \frac{4 \dot{B}}{B}$

We are going to solve these two differential equations (2.17) and (2.18) in Five unknowns $A, B, \lambda, \in$ and $\xi$. Here $\theta$ is a known quantity with the function of $A$ and $B$. In order to have solution, we assume three extra conditions. First we assume the relation between $A$ and $B$ (the scalar expansion $\theta$ is proportional to shear $\sigma$ assumed by Saha (2005)) as

$A=B^{2}$

On differentiating the above equation, we get

$\left(\frac{\ddot{A}}{A}-\frac{\dot{A}^{2}}{A^{2}}\right)=2\left(\frac{\ddot{B}}{B}-\frac{\dot{B}^{2}}{B^{2}}\right)$

After solving the above equations, we have

$\frac{\ddot{B}}{B}-\frac{\dot{B}^{2}}{B^{2}}=8 \pi B^{4}(\lambda+\epsilon)$

Assuming the second condition $\xi \theta=\mu$ (constant), i.e., the coefficient of bulk viscosity $\xi$ varies with the scalar expansion $\theta$, since the viscosity, being dissipative phenomenon, is believed to affect the expansion of the universe. Originally, this condition is assumed by Santos et al. (1985) and then many other researchers like Saha (2005) and Bali et al. (2007), etc.

The rest energy density $\in$ and the string tension density $\lambda$ for the composite matter of viscous fluid and dark energy can be taken together as

$\in=\epsilon_{V F}+\epsilon_{D E}, \quad \lambda=\lambda_{V F}+\lambda_{D E}$

and the energy momentum tensor equation (5.2.3) can be decomposed as

$T_{i}^{j}=\left(\epsilon_{V F}+\epsilon_{D E}\right) v_{i} v^{j}-\left(\lambda_{V F}+\lambda_{D E}\right) x_{i} x^{j}-\mu\left(g_{i}^{j}+v_{i} v^{j}\right)$ 
in which $\in_{D E}$ is the component of energy density corresponding to dark energy and $\lambda_{D E}$ is the component of string tension density corresponding to dark energy. The term $\in_{V F}$ is the rest energy density and $\lambda_{V F}$ is the string tension density corresponding to the viscous fluid.

\section{Model with Dark Energy}

\section{Quintessence Model}

Let us consider the case when the dark energy is given by a quintessence which obeys the equation of state $\lambda_{q}=\omega_{q} \in \epsilon_{q}, \omega_{q} \in[-1,0]$

In this quintessence model, the conservation law of energy momentum tensor, equation (can be written as $\dot{\epsilon}_{q}=\left(-2 \in_{q}+\lambda_{q}\right) \frac{2 \dot{B}}{B}+\mu \frac{4 \dot{B}}{B}$

Substitute the value of $\lambda_{q}=\omega_{q} \in_{q}$ from equation (3.1) in the above equation, we get

$\dot{\epsilon}_{q}=\left(-2 \in_{q}+\omega_{q} \in_{q}\right) \frac{2 \dot{B}}{B}+\mu \frac{4 \dot{B}}{B}$

which is a first order linear differential equation in $\epsilon_{q}$ and after solving above equation, we have

$\epsilon_{q}=\frac{2 \mu}{2-\omega_{q}}+\frac{c_{3}}{B^{\left(4-2 \omega_{q}\right)}}$

in which $c_{3}$ is the constant of integration.

Substituting the value of $\in_{q}$, in equation (3.1), we get

$$
\lambda_{q}=\omega_{q}\left(\frac{2 \mu}{2-\omega_{q}}+\frac{c_{3}}{B^{\left(4-2 \omega_{q}\right)}}\right)
$$

In particular $c_{3}=0$, we write

$$
\epsilon_{q}=\frac{2 \mu}{2-\omega_{q}} \quad \text { and } \quad \lambda_{q}=\omega_{q}\left(\frac{2 \mu}{2-\omega_{q}}\right)
$$

With these values of $\in_{q}$ and $\lambda_{q}$ (given by equation (3.2)), the equation (2.19) can be written as

$$
\frac{\ddot{B}}{B}-\frac{\dot{B}^{2}}{B^{2}}=\frac{2\left(1+\omega_{q}\right) \mu}{\left(2-\omega_{q}\right)} 8 \pi B^{4}
$$

(For $c_{3} \neq 0$, this differential equation (3.4), has complicated form and it is difficult to solve and hence we assumed $c_{3}=0$ ),

we have

$$
B=\left(c_{5} / \sqrt{l}\right)\left(\sinh \left(c_{4}-c_{5} t\right)\right)^{-1}
$$


where $c_{4}$ and $c_{5}$ are integration constants and $l=16 \pi\left(1+\omega_{q}\right) \mu /\left(2-\omega_{q}\right)=$ constant.

In view of relations $A=B^{2}$ and $B=C$, we write

$$
\begin{aligned}
& A=\left(c_{5}^{2} / l\right)\left(\sinh \left(c_{4}-c_{5} t\right)\right)^{-2} \\
& B=C=\left(c_{5} / \sqrt{l}\right)\left(\sinh \left(c_{4}-c_{5} t\right)\right)^{-1}
\end{aligned}
$$

The spatial volume $V$ is

$$
V=\left(c_{5}^{4} / l^{2}\right)\left(\sinh \left(c_{4}-c_{5} t\right)\right)^{-4}
$$

Here, it is to be noted that the equation of state parameter $\omega_{q} \neq-1$, since the scale factors $A, B$ and $C$ does not exist at $\omega_{q}=-1$. Thus the required dark energy quintessence model (for $\omega_{q} \neq-1$ ) is given by

$$
\begin{aligned}
d s^{2}=-d t^{2}+ & \left(c_{5}^{4} / l^{2}\right)\left(\sinh \left(c_{4}-c_{5} t\right)\right)^{-4} d x^{2} \\
& +\left(c_{5}^{2} / l\right)\left(\sinh \left(c_{4}-c_{5} t\right)\right)^{-2}\left(d y^{2}+d z^{2}\right)
\end{aligned}
$$

This quintessence dark energy model (3.8) for $\omega_{q} \neq-1$

The scale factors $A, B, C$ and the volume $V$ have the same nature in these two models and they behaving in hyperbolic sine function and contributed same geometrical and physical properties,

Thus it is observed that the geometry of quintessence dark energy model for $-1<\omega_{q}<0$ is matched with the geometry of viscous fluid model, for $1 \leq \gamma<0$.

From equations, the rest energy density $\in_{q}$, string tension density $\lambda_{q}$ and particle density $\in_{p}$ for quintessence dark energy model appeared with constant values and they are

$$
\begin{aligned}
& \epsilon_{q}=\frac{2 \mu}{2-\omega_{q}}, \quad \lambda_{q}=\omega_{q}\left(\frac{2 \mu}{2-\omega_{q}}\right) \text { and } \epsilon_{p}=\left(1-\omega_{q}\right)\left(\frac{2 \mu}{2-\omega_{q}}\right) \text {, for } \\
& \omega_{q} \neq-1
\end{aligned}
$$

independent of time $t$ and (non-zero) constant, for $\omega_{q} \neq-1$, which suggested the uniform distribution of dark energy matter and constant string phases in the universe.

The Hubble parameter $H$ and its directional's are 


$$
\begin{aligned}
H_{1} & =2 c_{5} \operatorname{coth}\left(c_{4}-c_{5} t\right), \quad H_{2}=H_{3}=c_{5} \operatorname{coth}\left(c_{4}-c_{5} t\right) \\
H & =\frac{4}{3} c_{5} \operatorname{coth}\left(c_{4}-c_{5} t\right)
\end{aligned}
$$

Other physical parameters are

$\theta=4 c_{5} \operatorname{coth}\left(c_{4}-c_{5} t\right), \quad \xi=\frac{\mu \tanh \left(c_{4}-c_{5} t\right)}{4 c_{5}}, \quad A=\frac{1}{8}$

$\sigma^{2}=\frac{1}{3} c_{5}^{2} \operatorname{coth}^{2}\left(c_{4}-c_{5} t\right), \quad q=-\frac{3}{4} \operatorname{sech}^{2}\left(c_{4}-c_{5} t\right)-1$

All the physical parameters like the Hubble parameter $H$, its directional's $H_{1}, H_{2}, H_{3}$, the scalar expansion $\theta$, the coefficient of bulk viscosity $\xi$, the magnitude of shear $\sigma$ and deceleration parameter $q$

\section{. 4. Conclusion}

The quintessence dark energy model for $-1<\omega_{q}<0$ (for suitable choice of constants) is in similar nature to the string viscous fluid model for $0<\gamma \leq 1$ in regards with its geometry and with physical significance of parameters (except energy density $\epsilon_{q}$, string tension density $\lambda_{q}$ and particle density) and also has hyperbolic inflationary solution. It is noticed that, in this dark energy quintessence models for $\omega_{q} \neq-1$, there is uniform distribution of dark matter and constant string phases in the universe. The dark energy quintessence model for $\omega_{q} \neq-1$ starts with open flat universe of special relativity and it is inflationary. The model filled with uniform distribution of dark energy matter and string phases of universe disappear, since string tension density is negative. The model expanding inflationary with accelerating phase. It is an anisotropize with shear and has constant nature of bulk viscosity.

\section{Refference}

[1] S.Perlmutter et al.Nature,vol. 391, pp. 51(1998).

[2] A.G. Riess et al., Astron. J., vol. 116, pp.1009 (1998).

[3] S.Perlmutter et al., Astrophys. J.,vol. 517, pp.565 (1999).

[4] R.A. Knop et al., Astrophys. J.,vol. 598, pp.102 (2003).

[5] A.G. Riess et al., Astrophys. J.,vol. 607,pp. 665 (2004).

[6] C.L. Bennett et al., Astron. Astrophys.Suppl. Ser., vol. 148, pp. 1 (2003).

[7] D.N. Spergel et al., Astron. Astrophys. Suppl. Ser.,vol. 148, pp. 175 (2003).

[8] M.Tegmark et al. (SDSS Collaboration), Phys. Rev.D,vol. 69, pp.103501(2004a).

[9] M.Tegmark et al. (SDSS Collaboration), Astrophys. J.,vol. 606, pp. 702 (2004b).
[10] U.Seljak et al.,Phys. Rev.D, vol.71, pp.103515 (2005).

[11] J.K.Adelman-McCarthy et al. (SDSS Collaboration) (2005).

[12] K.Abazajian et al. (SDSS Collaboration), (2003).

[13] K.Abazajian et al. (SDSS Collaboration) (2004).

[14] K. Abazajianet al. (SDSS Collaboration): (2004).

[15] B. Ratra, P.J.E. Peebles, Phys. Rev. D, vol. 37, pp. 3406 (1988).

[16] C.Wetterich, Nucl. Phys.,vol.B302, pp. 668 (1988).

[17] A.R Liddle, R.J Scherrer, Phys. Rev.D,vol. 59, pp. 023509 (1999).

[18] I.Zlatev,L.M. Wang, P.J. Steinhardt, Phys.

Rev. Lett., vol. 82, pp. 896 (1999). 
[19] R.R. Caldwell, Phys. Lett. B,vol. 545, pp. 23 (2002).

[20] R.R.Caldwell,M. Kamionkowski, N.N.

Weinberg, Phys. Rev. Lett., vol. 91, pp.071301(2003).

[21] S. Nojiri,S.D. Odintsov, Phys. Lett. B,vol. 562, pp.147 (2003).

[22] V.K. Onemli, R.P. Woodard, Phys. Rev. D, vol. 70, pp. 107301 (2004).

[23] M. R. Setare, J. Sadeghi, A.R. Amani, Phys. Lett. B,vol. 666, pp. 288 (2008).

[24] B. Feng,X.L. Wang, X.M. Zhang, Phys. Lett. B,vol. 607, pp. 35(2005).

[25] Z.K. Guo et al., Phys. Lett. B,vol. 608, pp.177 (2005).

[26] M.-Z. Li,B. Feng,X.-M. Zhang, J. Cosmol. Astropart. Phys.,vol. 002, pp. 0512 (2005).

[27] B. Feng,M. Li, Y.-S. Piao, X. Zhang, Phys. Lett. B,vol. 634, pp.101 (2006).

[28] W. Zhao, Y. Zhang, Phys. Rev. D, vol. 73, pp. 123509 (2006).

[29] M.R.Setare, J.Sadeghi, A.R. Amani, Phys. Lett. B,vol. 660, pp.299 (2008).

[30] J. Sadeghi, M.R. Setare, A. Banijamali, F. Milani, Phys. Lett. B,vol.662, pp.92 (2008).

[31] M.R. Setare, E.N. Saridakis, Phys. Lett. B,vol. 668, pp.177 (2008).

[32] M. R. Setare, E.N. Saridakis, arXiv:0807.3807 [hep-th].

[33] M.R. Setare, E.N. Saridakis, J. Cosmol. Astropart. Phys., vol. 0809, pp. 026 (2008).

[34] A.Y. Kamenshchik, U. Moschella, V. Pasquier, Phys. Lett. B, vol. 511, pp. 265 (2001). [35] M.R. Setare, Eur. Phys. J., vol. C52, pp. 689 (2007).

[36] M.R. Setare, Int. J. Mod. Phys.D,vol. 18, pp. 419 (2009).

[37] J. Kujat et al., Astrophys. J.,vol. 572, pp. 1 (2002).

[38] M. Bartelmann et al., New Astron. Rev.,vol. 49, pp. 199 (2005).
[39] R.Jimenez, New Astron. Rev., vol. 47, pp. 761 (2003).

[40] A.Das et al., Phys. Rev. D.,vol. 72, pp.

043528 (2005).

[41] M.S.Turner,M. White, Phys. Rev. D., vol. 56, pp. R4439 (1997).

[42] R.R.Caldwell et al., Phys. Rev. Lett., vol. 80, pp. 1582 (1998).

[43] A.R.Liddle, R.J. Scherrer, Phys. Rev. D, vol [44] N.Rosen, Plenum Press, London, pp. 273-94 (1977).

[45] T.M. Karade, Indian J. Pure-appl. Math, vol. 11(9), pp. 1202 (1980)

[46] M.Isrelit, Gen. Relativ. Gravit., vol. 13(7), pp. 681 (1981).

[47] D.R.K. Reddy, N. Venkateswara Rao, Astrophys. Space Sci., vol. 257, pp. 293 (1998).

[48] R. Bali, Anjali, Astrophys Space Sci.,vol. 302, pp. 201 (2006).

[49] R.Bali,S. Dave, Astrophys. Space Sci., vol. 288, pp. 503 (2003).

[50] R.Bali, R.D. Upadhaya, Astrophys. Space Sci. vol. 283, pp. 97 (2003).

[51] R. Bali, D. Singh, Astrophys. Space Sci., vol. 300, pp. 387 (2005).

[52] R. Bali, U.K. Pareek, Astrophys. Space Sci., vol. 312, pp. 305 (2007).

[53] S.D. Katore, R.S. Rane, Pramana J. Phys., vol. 67(2), pp. 237 (2006).

[54] G.S. Khadekar, S.D. Tade, Astrophys. Space Sci. (1998) DOI 10.1007\S10509 - 007- 9410-2.T. [55] M.S. Borkar, S.S. Charjan, IJAM, vol. 22(3), pp. 445, (2009).

[57] M.S. Borkar, S.S. Charjan, J. Ind. Acad. Math, vol. 32(1), (2010).

[58] M.S. Borkar, S.S. Charjan, An Int. J. AAM, vol. 5(1), pp. 96109 (2010).

[59] M.S. Borkar, S.S. Charjan, An Int. J. AAM , vol. 5(2), pp. 1660 (2010).

[60] N.P. Gaikwad et al.: Chin. Phys.

Lett.,vol.28(8), pp. 089803 (2011) 